\title{
The incomputable reality
}

\author{
The natural world's interconnectivity should inspire better \\ models of the Universe, says Barry Cooper.
}

A lan Turing put bounds on what is computable in a famous 1936 paper $^{1}$. The Turing machines he presented implement finite algorithms, handling data coded as real numbers. They are deterministic, but give some bizarre results. You can build a universal machine that can simulate any other Turing machine. But not every question you can ask of it has a computable answer: you cannot predict, for example, whether it ever spits out a given number or series of numbers.

By coincidence, our Newtonian view of physics faltered at about the same time as our computable view of mathematics. Lingering problems in classical physics, such as the unpredictable trajectories of three bodies following a collision, may involve incomputability. Albert Einstein's theory of general relativity opens up a new world of computation with exotic objects such as spinning black holes. Quantum mechanics tells us that measurements are inherently uncertain.

The concept of computability is basic to modern science, from quantum gravity to artificial intelligence. It is also relevant in the everyday world, where it is useful to distinguish problems that are merely difficult to compute in practice from those that are intrinsically impossible with any machine. Incomputability should trouble economists, because breakdowns of control in chaotic markets can wreak havoc.

But disciplinary boundaries are preventing us from getting a full view of its role. Cosmetic differences may hide revealing parallels.

\section{EMERGENT PHENOMENA}

Turing was interested in the mathematics of computing and also in its embodiment - the material environment that houses it. This theme links all of his work, from machines to the brain and morphogenesis. Although many mathematicians and software engineers today see it as irrelevant, embodiment is key to explaining the physical world.

Take turbulence: a river swollen by recent rain occasionally erupts into surprising formations that we would not expect from the basic dynamics of the water flow. The reason is coherence - non-local connectivity affects the water's motion. Turbulence, and

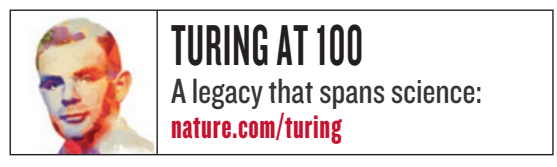

other 'emergent' nonlinear phenomena, may not be computable with a Turing machine. Zebra stripes and tropical-fish patterns, which Turing described in 1952-54 with his differential equations for morphogenesis, arise similarly.

Even in nonlinear systems, such highorder behaviour is causal - one phenomenon triggers another. Levels of explanation, from the quantum to the macroscopic, can be applied. But modelling the evolution of the higher-order effects is difficult in anything other than a broad-brush way. Such problems infiltrate all our models of the natural world.

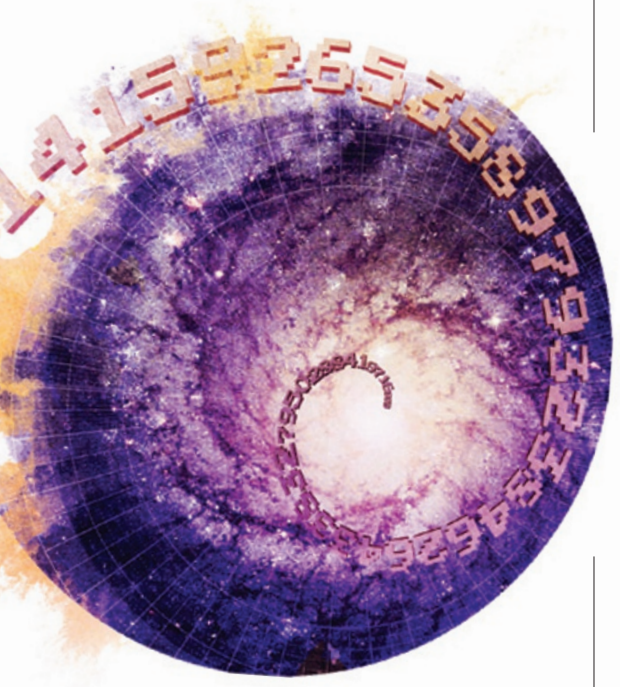

The Universe is like that turbulent stream — its behaviour as a whole guided by myriad connections at various scales. It has many emergent levels of causality, bridged by phase transitions. The mechanistic structure that science deals with so well, and its invariant laws, are hard to explain in terms of the quantum level. Biology emerges from the quantum world, but is not computable from it. We are part of an organic whole fragmented but coherent.

Across these boundaries, higher-level relations can feed back into lower ones. But looking up from a lower level, the causality will not be computable. For example, the uncertainty principle prevents the quantum world from fully describing the state of a particle at any instant. A measurement produces a full description, but we cannot compute how it does it. In Turing's world, a description of reality is not always enough for a computable prediction.

Nature presents us with new ways of computing, from the Universe to the brain. Turing went on to build logical hierarchies to better understand real-world computation, which includes intuitive or unpredictable leaps ${ }^{2}$. Researchers experimenting with intelligent machines today see the possibilities in such an approach. But problems of control of higher-order behaviours still present formidable challenges to implementing it.

\section{BRIDGE BUILDING}

It took nature millions of years to build a human brain. Meanwhile, we have to live with the stupidity of purely algorithmic processes. We need to embrace more experimental approaches to computation, and a renewed respect for embodied computing - as anticipated in Turing's late work in the 1950 s on artificial intelligence and morphogenesis.

Bridges between mathematicians and physicists are important if we are to do this. It is a long time since Kurt Gödel and Albert Einstein chatted in the halls of Princeton University in New Jersey. Mathematicians can bring to the table Turing's model of basic causal structure. This would help physicists to discover more complete descriptions of the Universe - making redundant Hugh Everett's many-worlds interpretation and related multiverse hypotheses - and fix the arbitrariness of parts of the standard model of particle physics.

Samson Abramsky, a computer scientist at the University of Oxford, UK, recently asked: "Why do we compute?" Turing computation does not create anything that is not there already in the initial data. Can information increase in computation?

If we look at the world with new eyes, allowing computation full expression, we may come to startling conclusions.

Barry Cooper is in the School of Mathematics, University of Leeds, Leeds LS2 9JT, UK. e-mail:pmt6sbc@leeds.ac.uk

1. Turing, A. M. Proc. Lond. Math. Soc. s2-42, 230-265 (1936-37).

2. Turing, A. M. Proc. Lond. Math. Soc. s2-45, 161-228 (1939). 